\title{
Polarization tomography of metallic nanohole arrays
}

\author{
Erwin Altewischer, Cyriaque Genet, Martin P. van Exter, and J. P. Woerdman
}

Huygens Laboratory, Leiden University, P.O. Box 9504, 2300 RA Leiden, The Netherlands

Paul F. A. Alkemade

Kavli Institute of Nanoscience, Delft University of Technology, Rotterdamseweg 137, 2628 AL Delft, The Netherlands

Arjan van Zuuk and Emile W. J. M. van der Drift

Delft Institute of Microelectronics and Submicrontechnology, Delft University of Technology, P.O. Box 5053, 2600 GB Delft, The Netherlands

Received August 4, 2004

\begin{abstract}
We report polarization tomography experiments on metallic nanohole arrays with square and hexagonal symmetry. As a main result we find that a fully polarized input beam is partly depolarized after transmission through a nanohole array. This loss of polarization coherence is found to be anisotropic; i.e., it depends on the polarization state of the input beam. The depolarization is ascribed to a combination of two factors: (i) the nonlocal response of the array as a result of surface-plasmon propagation and (ii) the non-plane-wave nature of a practical input beam. (C) 2005 Optical Society of America
\end{abstract}

OCIS codes: $230.3990,240.6680,260.3910$.

Currently there is much interest in the optical properties of thin metal films perforated with arrays of subwavelength holes, or nanohole arrays. The optical transmission of these arrays shows a strongly peaked spectrum with anomalously large transmission peak values; this is usually ascribed to resonant excitation of propagating surface electromagnetic waves or surface plasmons (SPs). ${ }^{1-3}$ In this Letter we focus on the polarization properties of the anomalous transmission and show that these are strongly influenced by the propagating nature of the SPs.

So far, polarization properties of nanohole arrays have been studied in a limited context: a beam with a given uniform state of polarization $\left(\mathrm{SOP}_{\text {in }}\right)$ is transformed by an anisotropic array or an isotropic array with nonspherical holes into a different uniform state of output polarization $\left.\left(\mathrm{SOP}_{\text {out }}\right)\right)^{4-6}$ This corresponds to a mapping of the Poincaré sphere onto itself; for instance, a rectangular array or a square array with elliptical holes acts as a birefringent and (or) a dichroic element that may convert a linear SOP into an elliptical SOP, conserving polarization coherence. In the present Letter we focus instead on cases where the degree of polarization (DOP) is reduced, $\mathrm{DOP}_{\text {out }}<\mathrm{DOP}_{\text {in }}$, corresponding to a reduction in radius and, in general, a deformation of the Poincaré sphere. ${ }^{7,8}$ To underline this point we have chosen for our experiments square and hexagonal arrays, i.e., arrays that, for symmetry reasons, ${ }^{9}$ cannot modify the SOP for plane-wave illumination at normal incidence. As we will show, depolarization occurs when two (quite common) conditions are simultaneously fulfilled: (i) the response of the array is nonlocal because of SP propagation, and (ii) the input beam is not a plane wave [but, e.g., a Gaussian beam, with a finite numerical aperture (NA)].

In general, depolarization occurs when an optical system acts nonuniformly on polarization within the (spatial or temporal) bandwidth of the incident wave, thereby coupling polarization to other degrees of freedom. Experimentally, a study of depolarization requires a measurement of the Mueller matrix by a tomographic method. ${ }^{7,8}$ Here we report such polarization-tomography experiments on nanohole arrays and interpret the results in the context of SP propagation.

We start by recapitulating the essence of our theoretical model. ${ }^{9}$ The input and output optical fields of the array are related via a nonlocal linear response as $\mathbf{E}_{\text {out }}(\mathbf{r}, \omega)=\int t\left(\mathbf{r}-\mathbf{r}^{\prime}, \omega\right) \mathbf{E}_{\text {in }}\left(\mathbf{r}^{\prime}, \omega\right) \mathrm{d} \mathbf{r}^{\prime}$. In the far field, or Fourier domain, this is equivalent to $\mathbf{E}_{\text {out }}\left(\mathbf{k}_{t}, \omega\right)=t\left(\mathbf{k}_{t}, \omega\right) \mathbf{E}_{\text {in }}\left(\mathbf{k}_{t}, \omega\right)$, where $\mathbf{k}_{t}$ is the transverse wave-vector component; the output $\mathbf{k}_{t}$ is equal to the input $\mathbf{k}_{t}$ for the zeroth-order transmission. If the nonlocal response depends on polarization, the four elements of the $2 \times 2$ transmission tensor $t\left(\mathbf{k}_{t}, \omega\right)$ will exhibit a different angular dependence and the output field $\mathbf{E}_{\text {out }}\left(\mathbf{k}_{t}, \omega\right)$ will have a spatially dependent polarization even for a polarization-pure input field. ${ }^{10}$ After spatial integration this transmission process can be captured in the simple relation $S_{\text {out }}=M S_{\text {in }}$, which relates the input and output Stokes vectors through the $4 \times 4$ Mueller matrix $M$. For ideal square and hexagonal arrays the Mueller matrix has been predicted to be diagonal (no mixing of Stokes parameters). ${ }^{9}$ For hexagonal arrays, the additional symmetry relation $M_{11}=M_{22}$ holds.

The magnitudes of the diagonal elements $M_{00}, M_{11}$, $M_{22}$, and $M_{33}$ depend on the dimensionless product of the SP propagation length and the wave-vector spread of the input beam, $l_{\mathrm{SP}} \Delta k_{t}$. A full theoretical description thereof would require a microscopic model; however, from physical considerations it can be seen that an appreciable deviation of $M_{i i} / M_{00}$ from 1 requires $l_{\mathrm{SP}} \Delta k_{t}>1$. In any case there will be no depolarization if either there are no propagating waves $\left(l_{\mathrm{SP}}=0\right)$ or there is plane-wave illumination 
$\left(\Delta k_{t}=0\right)$; both propagation and a wave-vector spread are necessary. This statement holds for hole arrays of any symmetry (i.e., not only for the square and hexagonal arrays used in our experiment).

To measure the Mueller matrices we use a linearly polarized titanium:sapphire laser at a wavelength of $810 \mathrm{~nm}$, which is the approximate resonance wavelength of the hole arrays (see Fig. 1), to illuminate the input lens of a symmetric telescope. After transmission through a hole array positioned at the focus of the telescope, the light is imaged onto a photodiode. The SOP of the incident light is set by a rotatable quarter- or half-wave plate in front of the first lens. The Stokes parameters at the output are measured with a rotatable quarter-wave plate and a polarizer positioned in front of the photodiode. The polarization isotropy of all optical components was checked by measuring the Mueller matrices of the setup in the absence of hole arrays. These matrices were practically equal to the identity matrix, with individual elements deviating by no more than 0.02 (typically 0.008 ).

Our arrays were fabricated in Au films on glass substrates. We used a square array made with electronbeam lithography, with a lattice spacing of $700 \mathrm{~nm}$ and a nominal hole diameter of $200 \mathrm{~nm}$, and a hexagonal array made with ion-beam milling, with a lattice spacing of $886 \mathrm{~nm}$ and a nominal hole diameter of $200 \mathrm{~nm}$. Transmission spectra of both arrays under almost plane-wave illumination at normal incidence are shown in Fig. 1. Both arrays show a resonance wavelength of $810 \mathrm{~nm}$; the polarization experiments were performed at this wavelength. These resonances correspond to SPs propagating in the $( \pm 1, \pm 1)$ direction at the metal-glass interface for the square array, and the (sixfold degenerate) $(1,0,0)$ direction at the metal-air interface for the hexagonal array (the labeling is with respect to the reciprocal lattice vectors). ${ }^{11}$ The linewidths are 40 and $25 \mathrm{~nm}$ for the square and hexagonal arrays, respectively, from which we estimate $l_{\mathrm{SP}} \approx 2 \mu \mathrm{m}$ and $l_{\mathrm{SP}} \approx 4 \mu \mathrm{m}$, respectively. ${ }^{12}$ The difference in $l_{\mathrm{SP}}$ values is probably caused by both the different arrays structures and the different resonant surfaces (less damping on the airmetal interface than on the glass-metal interface).

We have characterized the arrays with almost plane-wave illumination (NA of 0.01 or $\Delta k_{t} \approx 0.08 \mu \mathrm{m}^{-1}$ ) and focused illumination (for up to a NA of $\approx 0.15$ or $\Delta k_{t} \approx 1.2 \mu \mathrm{m}^{-1}$ ). Figure 2 shows the dependence of the diagonal elements of the Mueller matrix on the NA of the incident light for both arrays. The figure shows that, for the case of almost plane-wave illumination (NA $\approx 0$ ), the $M_{i i}$ values are close to 1 for both arrays. There is no depolarization, as $\Delta k_{t} \approx 0$. However, for increasing wave-vector spread, or decreasing spot size on the array, the depolarizing effect of the arrays quickly increases. Furthermore, the depolarization is clearly anisotropic, i.e., the amount of depolarization depends on the input SOP, because $M_{i i}$ are not all equal. For the hexagonal array the depolarization sets in faster with increasing NA than for the square array, because of the smaller resonance linewidth (larger $l_{\mathrm{SP}}$ ) of the hexagonal array.
The effect of the different array symmetries on the curve shapes are prominent. For the square array [Fig. 2(a)], the observed inequality $M_{22}>M_{11}$ shows that there is less depolarization for an input polarization along either of the array diagonals than for a polarization along the main axes. This observation is consistent with the $( \pm 1, \pm 1)$ propagation directions of the resonantly excited SPs on the metal-glass interface; as SPs are mainly longitudinally polarized, they preserve polarization along their propagation direction. However, the deviation of $M_{22}$ from 1 indicates that the $( \pm 1, \pm 1)$ SPs are not the only SPs involved in the transmission process; other (nonresonant) SPs on both surface apparently contribute. ${ }^{10}$ For the hexagonal array [Fig. 2(b)] the theoretical equality $M_{11}=M_{22}$ also holds quite well. For both arrays, $M_{33}$ is the smallest diagonal element. This shows that depolarization is most dramatic for circularly polarized light, a case that, to our knowledge, has not been studied before. We note that $M_{33} \approx M_{11}+M_{22}-1$ for both arrays; this follows from an extension of the symmetrybased theory used here by explicit modeling of SP propagation..$^{14}$

The full Mueller matrices for both arrays are shown in Table 1. The diagonal elements (marked with boxes in the table) conform to the discussion given above. The off-diagonal elements of a perfectly symmetric square or hexagonal array should theoretically

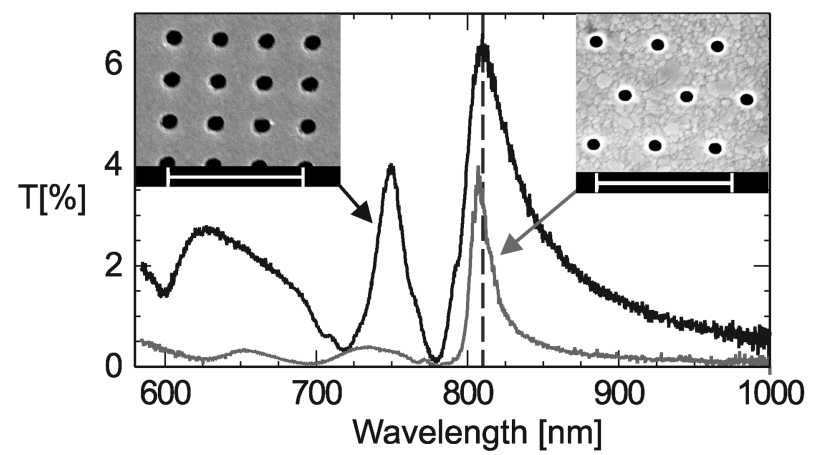

Fig. 1. Transmission spectra of nanohole arrays under almost plane-wave illumination at normal incidence for the square and hexagonal array. The dashed vertical line indicates the resonance wavelength of $810 \mathrm{~nm}$ used in the experiments. The insets show scanning electron-beam micrographs of the arrays, with $2-\mu \mathrm{m}$-scale bars.
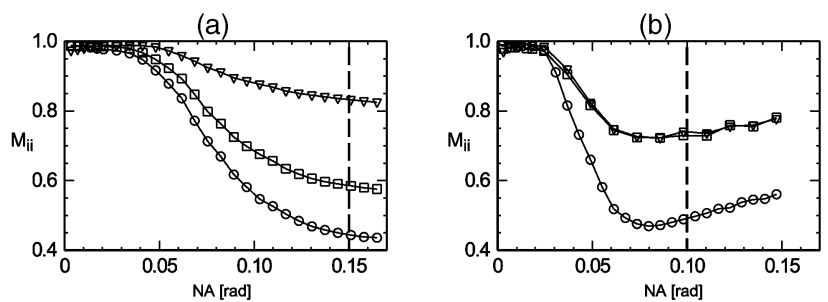

Fig. 2. Diagonal elements $M_{i i}$ of the Mueller matrix, normalized to $M_{00}$, as a function of the NA of the incident light beam on the (a) square and (b) hexagonal hole array. Squares, $M_{11}$; triangles, $M_{22}$; circles, $M_{33}$; dashed vertical lines indicate the NAs at which the full Mueller matrices were measured (see Table 1). 
Table 1. Mueller Matrices Measured Under Four Different Conditions ${ }^{a}$

\begin{tabular}{|c|c|c|c|c|}
\hline \multirow[t]{2}{*}{ Measurement } & \multicolumn{4}{|c|}{ Mueller Matrix $M$} \\
\hline & 1.00 & 0.00 & -0.02 & 0.00 \\
\hline \multirow{3}{*}{$\begin{array}{l}\text { Square array } \\
\text { NA } 0.01 \\
l_{\mathrm{SP}} \Delta k_{t} \approx 0.2\end{array}$} & -0.02 & 1.01 & -0.01 & 0.00 \\
\hline & 0.00 & 0.00 & 1.01 & -0.01 \\
\hline & 0.00 & 0.00 & 0.02 & 0.99 \\
\hline \multirow{4}{*}{$\begin{array}{l}\text { Square array } \\
\text { NA } 0.15, \\
l_{\mathrm{SP}} \Delta k_{t} \approx 2\end{array}$} & 1.00 & 0.01 & -0.02 & 0.01 \\
\hline & 0.01 & 0.55 & -0.04 & -0.03 \\
\hline & -0.04 & 0.02 & 0.84 & 0.01 \\
\hline & -0.01 & 0.01 & 0.01 & 0.41 \\
\hline \multirow{4}{*}{$\begin{array}{l}\text { Hexagonal array } \\
\text { NA } 0.01, \\
l_{\mathrm{SP}} \Delta k_{t} \approx 0.3\end{array}$} & 1.00 & 0.06 & -0.14 & 0.00 \\
\hline & 0.04 & 1.00 & 0.00 & -0.07 \\
\hline & -0.12 & 0.01 & 1.01 & -0.03 \\
\hline & $\overline{0.00}$ & $\underline{0.06}$ & 0.02 & 0.97 \\
\hline \multirow{4}{*}{$\begin{array}{l}\text { Hexagonal array } \\
\text { NA } 0.10 \\
l_{\mathrm{SP}} \Delta k_{t} \approx 3\end{array}$} & 1.00 & 0.03 & -0.11 & 0.00 \\
\hline & 0.02 & 0.78 & 0.00 & -0.08 \\
\hline & -0.13 & 0.01 & 0.78 & 0.00 \\
\hline & 0.00 & $\underline{0.06}$ & 0.02 & 0.51 \\
\hline
\end{tabular}

${ }^{a}$ Normalization constants (absolute intensity transmission for unpolarized light) of the matrices are $1.4 \%$ (hexagonal NA 0.01), $0.47 \%$ (hexagonal NA 0.10 ), $7.1 \%$ (square NA 0.01 ), and $2.2 \%$ (square NA 0.15)

be zero. For our square array they are indeed relatively small and do not show any systematic behavior. For the hexagonal array, however, these elements are much larger, both for plane-wave and focused illumination. Furthermore, the off-diagonal elements have a clear pattern and similar values in both cases, where the odd off-diagonal elements $M_{02}, M_{20}, M_{13}$, and $M_{31}$ (underlined in Table 1) are substantially larger than the others. The presence of this pattern was also checked for an intermediate NA of 0.03 . The pattern is compatible with a birefringent and (or) a dichroic $45^{\circ}$ axis, which is apparently due to array errors, such as a spatially variant lattice spacing or ellipticity of the holes. These errors could be created by alignment errors or even intrinsic imperfections in the ion-beam optics (astigmatism and deflection errors). Our work shows that polarization tomography provides for sensitive diagnostics of array symmetry imperfections.

From a general perspective, Mueller tomography is a purely phenomenological tool to characterize the polarization behavior of optical systems. Still, it can give new insight into the physical mechanisms that are active in hole arrays, in our case SP propagation. It would, for instance, be interesting to perform
Mueller tomography on metal hole arrays in the (sub)millimeter-wave regime, as SPs propagate much farther in this part of the spectrum, which is expected to increase the depolarization. Another area of interest is the connection between classical polarization properties and entanglement degradation. ${ }^{13}$

In conclusion, we have demonstrated surprising consequences of SP propagation for the polarization behavior of nanohole arrays. The nonlocality of the array response forms an essential ingredient of the physics of these intriguing devices.

This work was supported by the Stichting voor Fundamenteel Onderzoek der Materie (FOM); partial support was provided by the European Union under the Information Society Technologies Active Teleportation and Entangled State Information Technology Contract. E. Altewischer's e-mail address is erwin@molphys. leidenuniv.nl.

\section{References}

1. T. W. Ebbesen, H. J. Lezec, H. F. Ghaemi, T. Thio, and P. A. Wolff, Nature 391, 667 (1998).

2. H. F. Ghaemi, T. Thio, D. E. Grupp, T. W. Ebbesen, and H. J. Lezec, Phys. Rev. B 58, 6779 (1998).

3. H. Raether, Surface Plasmons (Springer-Verlag, Berlin, 1988).

4. J. Elliott, I. I. Smolyaninov, N. I. Zheludev, and A. V. Zayats, Opt. Lett. 29, 1414 (2004).

5. K. J. K. Koerkamp, S. Enoch, F. B. Segerink, N. F. van Hulst, and L. Kuipers, Phys. Rev. Lett. 92, 183901 (2004).

6. R. Gordon, A. G. Brolo, A. McKinnon, A. Rajora, B. Leathem, and K. L. Kavanagh, Phys. Rev. Lett. 92, 037401 (2004). Unfortunately, the authors employed the word "depolarization" in an improper way, using it to describe a change of a spatially uniform SOP.

7. R. M. A. Azzam and N. M. Bashara, Ellipsometry and Polarized Light (North-Holland, Amsterdam, 1987).

8. F. Le Roy-Brehonnet and B. Le Jeune, Prog. Quantum Electron. 21, 109 (1997).

9. C. Genet, E. Altewischer, M. P. van Exter, and J. P. Woerdman, "Depolarization induced by subwavelength metal hole arrays," November 28, 2003, arXiv:physics/ 0311137, http://xxx.lanl.gov.

10. E. Altewischer, M. P. van Exter, and J. P. Woerdman, J. Opt. Soc. Am. B 20, 1927 (2003).

11. T. Thio, H. F. Ghaemi, H. J. Lezec, P. A. Wolff, and T. W. Ebbesen, J. Opt. Soc. Am. B 16, 1743 (1999).

12. D. S. Kim, S. C. Hohng, V. Malyarchuk, Y. C. Yoon, Y. H. Ahn, K. J. Yee, J. W. Park, J. Kim, Q. H. Park, and C. Lienau, Phys. Rev. Lett. 91, 143901 (2003).

13. E. Altewischer, M. P. van Exter, and J. P. Woerdman, Nature 418, 304 (2002).

14. E. Altewischer, M. P. van Exter, and J. P. Woerdman are preparing a manuscript to be called "Analytic model of optical depolarization in square and hexagonal nanohole arrays." 